\title{
Assessment of retinal and optic disc vascular perfusion density in retinitis pigmentosa patients by optical coherence tomography angiography
}

Katarzyna Baltaziak, Lucyna Baltaziak, Rashed Mustafa Nazzal@ ( Anna Woźniak, Robert Rejdak (1)

Department of General Ophthalmology, Medical University of Lublin, Lublin, Poland

\begin{abstract}
BACKGROUND: The aim of the study was to evaluate the vascular flow density measured by optical coherence tomography angiography in patients with retinitis pigmentosa and to compare it with normal subjects.

MATERIAL AND METHODS: This was a cross-sectional case control study employing 16 eyes of eight patients with retinitis pigmentosa and 16 eyes of eight healthy subjects. Optical coherence tomography angiography was performed in all subjects. The macula was imaged with a $3 \times 3 \mathrm{~mm}$ scan, whereas for the optic nerve head, a $4.5 \times 4.5 \mathrm{~mm}$ scan was taken. Macular and optic disc vascular flow perfusion densities were measured. In addition, the FAZ area was calculated. All data were compared in the two groups.

RESULTS: The vascular flow perfusion density in the macular and in the optic nerve head was significantly lower in the retinitis pigmentosa group when compared with the control group. On the other hand, the foveal avascular zones area was not significantly different from controls.

CONCLUSION: Patients with retinitis pigmentosa show a decreased macular and optic nerve head perfusion density compared with healthy subjects

KEY WORDS: retinitis pigmentosa; OCTA; angiography; OCT; optical coherence tomography
\end{abstract}

Ophthalmol J 2019; Vol. 4, 100-104

\section{INTRODUCTION}

Retinitis pigmentosa (RP) is a progressive genetically heterogeneous group of diseases characterised by the primary dysfunction of rod photoreceptors and secondary impairment of cones in later stages of diseases. Usually patients present complaining of the triad of nyctalopia, progressive visual field loss, and reduced visual acuity. It is considered to be the most common form of inherited retinal dystrophy [1]. Retinitis pigmentosa has characteristic features on fundus examination: attenuation of retinal blood vessels, waxy looking pale optic disc, atrophy of the retinal pigment epithelium (RPE), and peripheral bone spicule pigmentation [2].

Optical coherence tomography angiography (OCTA) is a new, rapid, noninvasive, safe, reliable, and easily performed imaging method that provides high depth-resolved data about retinal and choroidal blood flow in many vascular diseases [3]. Some of the previously published cross-sectional OCTA studies have demonstrated that the superficial and deep capillary plexus perfusion densities are de- 
creased in RP patients; in addition, the FAZ area was demonstrated to be enlarged [4].

The aim of this study was to investigate the retinal circulation of patients with RP using OCTA to determine whether the flow areas were reduced in patients with RP in comparison to normal subjects.

\section{MATERIAL AND METHODS}

All study procedures were done after taking informed consent as per Medical University of Lublin policy. The study followed the Declaration of Helsinki guidelines. A review of records of $80 \mathrm{pa}-$ tients with confirmed clinical diagnosis of retinitis pigmentosa was conducted at the Department of General Ophthalmology at the Medical University of Lublin. The clinical diagnosis was made based on presenting symptoms, family history, fundus examination, visual field, and electroretinography (ERG) and subsequently supported by clinical imaging and/or genetic testing. The inclusion criteria for this study were a diagnosis of RP and adequate fixation to allow for high-quality imaging. Ocular examinations included a slit-lamp and dilated fundoscopy examination, best corrected visual acuity (BCVA) by decimal Snellen chart, and OCTA. We compared the vascular perfusion density in several perspectives: first we compared the perfusion density in the whole macula in each eye, and then the integrated software in the OCTA subdivided the macular region into two parts - superior half and inferior half. Each half was studied separately. The exclusion criteria included patients affected by any other ocular disorder (e.g. glaucoma) and eyes with poor OCTA images that resulted in a signal strength index lower than 7 out of 10 . We ended up with eight patients out of 80 .

\section{STATISTICAL ANALYSIS}

The statistical analysis was performed using Microsoft Office Excel 2010. Statistical analysis included descriptive statistics for demographics, Snellen chart BCVA (decimal), and OCTA measurements (macular vascular perfusion density for the superficial capillary plexus [SCP] and deep capillary plexus [DCP], FAZ area for the SCP and DCP, and choriocapillaris blood flow) for both the macula and the optic disc. One-sample Student's t-test was used to determine if there was a significant difference in readings. In all of the statistical analyses, we defined statistical significance as a p-value of less than 0.05.

\section{CLINICAL EXAM}

Imaging took place after proper pupil dilation with tropicamide eye drops $(1 \%)$.

OCTA $3 \times 3 \mathrm{~mm}$ scans centred on the fovea and $4.50 \mathrm{~mm}$ of the optic disc were obtained using the AngioVue (Optovue Inc., Fremont, CA, USA). This device has a scanning speed of 70,000 A-scans per second and an optical axial resolution of 5 microns and transverse resolution of 15 microns with an axial depth of up to 3 microns and wavelength-scanning light of $840 \mathrm{~nm}$ equipped with Split-Spectrum Amplitude-Decorrelation Angiography (SSADA) algorithm. Automated segmentation of full-thickness retina scans into SCP, DCP, and choriocapillaris by the machine was performed. The SCP is bounded by the internal limiting membrane and the inner plexiform layer, containing vasculatures from the nerve fibre, ganglion cell, and inner plexiform layers. The DCP is bounded by the inner plexiform and outer plexiform layers, containing vasculatures from the inner nuclear and outer plexiform layers

\section{RESULTS}

In this cross-sectional case-control study, 16 eyes of eight patients with retinitis pigmentosa were employed and compared with 16 eyes of eight patients with age- and sex-matched controls. There were four males and four females in each group with an average (mean) age of 44 and 42 years, respectively (Tab. I). All patients were Caucasians. The mean visual acuity as measured by Snellen chart (decimal scale) was 1.0 for controls and 0.63 for retinitis pigmentosa patients. Upon comparison of the perfusion density in the whole macula in each eye (Tab. I), there was a significant drop of vascularity in RP group in comparison to the control group ( $p>0.05$ ). In addition, the superior half and inferior half of the macular area showed significant perfusion drop in RP patients in comparison to controls also ( $\mathrm{p}>0.05)$. All of these values were taken for both SCP and DCP, and the results were significant for both layers. Although the foveal avascular zone (FAZ) areas were enlarged in RP patients at both the SCP and DCP levels, these figures failed to achieve statistical significance in our study $(p<0.05)$ (Fig. 1). On the other hand, choriocapillaris blood flow values were not statistically significant from the values of the control group. Then a comparison of vascular perfusion density overlying the optic disc among the two groups was performed, revealing a significant 


\begin{tabular}{|c|c|c|c|}
\hline & Control & Patients & p-value \\
\hline Number of eyes (patients) & $16(8)$ & $16(8)$ & \\
\hline Gender (Male:Female) & $4: 4$ & $4: 4$ & \\
\hline Mean age (years) & 42 & 44 & 0.35 \\
\hline $\begin{array}{l}\text { Best corrected visual acuity } \\
\text { Snellen chart (decimal) }\end{array}$ & $1.0(\mathrm{OD} 1.0, \mathrm{OS} 1.0)$ & 0.63 (OD 0.650, OS 0.625) & $\begin{array}{l}\text { OD } 0.004 \\
\text { OS } 0.005\end{array}$ \\
\hline \multicolumn{4}{|c|}{ Perfusion density (mean value, \%) } \\
\hline The whole image & $\begin{array}{l}\text { SCP } 46.8 \\
\text { DCP } 48.1\end{array}$ & $\begin{array}{l}\text { SCP } 37.9 \\
\text { DCP } 37.6\end{array}$ & $\begin{array}{l}0.0008 \\
0.00006\end{array}$ \\
\hline Superior hemi & $\begin{array}{l}\text { SCP } 46.8 \\
\text { DCP } 48.8\end{array}$ & $\begin{array}{l}\text { SCP } 38.2 \\
\text { DCP } 37.4\end{array}$ & $\begin{array}{l}0.0008 \\
0.0001\end{array}$ \\
\hline Inferior hemi & $\begin{array}{l}\text { SCP } 46.7 \\
\text { DCP } 47.5\end{array}$ & $\begin{array}{l}\text { SCP } 37.5 \\
\text { DCP } 37.9\end{array}$ & $\begin{array}{l}0.0009 \\
0.00008\end{array}$ \\
\hline FAZ area (mean value, $\mathrm{mm}^{2}$ ) & $\begin{array}{l}\text { SCP } 0.25 \\
\text { DCP } 0.25\end{array}$ & $\begin{array}{l}\text { SCP } 0.32 \\
\text { DCP } 0.26\end{array}$ & $\begin{array}{l}0.2595 \\
0.4755\end{array}$ \\
\hline CBF (mean value, \%) & $\begin{array}{l}\text { OS } 0.54 \\
\text { OD } 0.50\end{array}$ & $\begin{array}{l}\text { OS } 0.68 \\
\text { OD } 0.68\end{array}$ & $\begin{array}{l}0.1557 \\
0.0638\end{array}$ \\
\hline \multicolumn{4}{|c|}{ Optic disc vascular density (mean value \%), RPC } \\
\hline Whole image & $\begin{array}{l}\text { OS } 49.5 \\
\text { OD } 49.9\end{array}$ & $\begin{array}{l}\text { OS } 45.7 \\
\text { OD } 45.8\end{array}$ & $\begin{array}{l}\text { OS } 0.02043 \\
\text { OD } 0.00853\end{array}$ \\
\hline Inside the disc & $\begin{array}{l}\text { OS } 52.1 \\
\text { OD } 53.3\end{array}$ & $\begin{array}{l}\text { OS } 47.7 \\
\text { OD } 49.7\end{array}$ & $\begin{array}{l}\text { OS } 0.04106 \\
\text { OD } 0.02249\end{array}$ \\
\hline Peripapillary & $\begin{array}{l}\text { OS } 52.8 \\
\text { OD } 52.7\end{array}$ & $\begin{array}{l}\text { OS } 44.2 \\
\text { OD } 43.7\end{array}$ & $\begin{array}{l}\text { OS } 0.00301 \\
\text { OD } 0.00102\end{array}$ \\
\hline
\end{tabular}

$\mathrm{OD}$ — oculus dexter, $\mathrm{OS}$ - oculus sinister; $S C P$ - superficial capillary plexus; $\mathrm{DCP}$ - deep capillary plexus; FAZ — foveal avascular zone; $C B F$ — choriocapillaris blood flow; RPC — radial peripapillary capillary

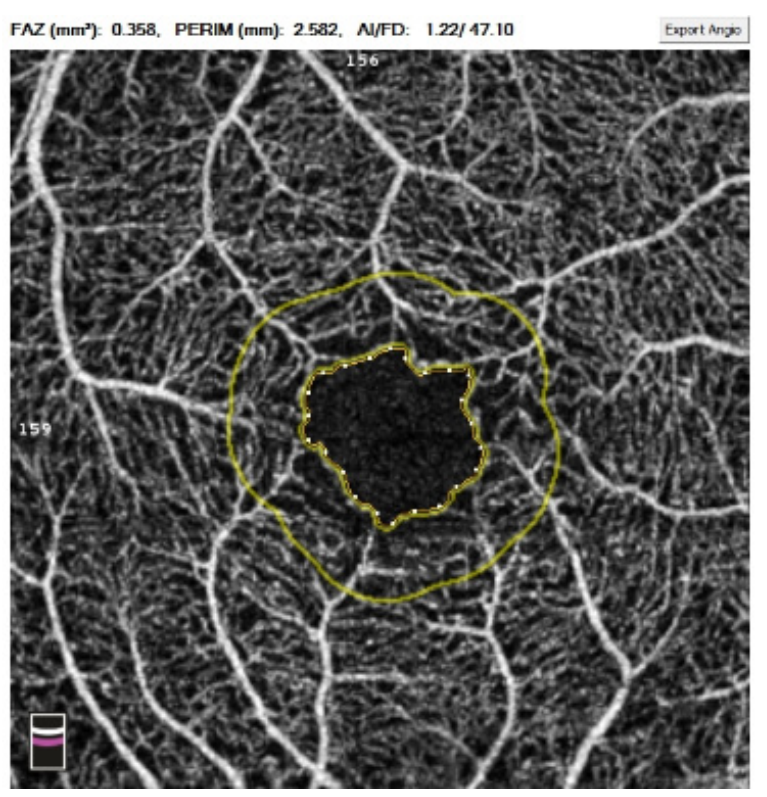

FIGURE 1. One of the patient's foveal avascular zone (FAZ) area

drop in all parameters in RP patients: whole image, inside the disc, and peripapillary (Fig. 2).

\section{DISCUSSION}

There has always been some speculation about the reduction of retinal vascular supply in patients with $\mathrm{RP}$ in comparison with healthy subjects. In the era of OCTA, there have been few reports confirming this theory in a non-invasive, fast, and reliable technique. We carried out this study to report our results and to see if they are in general agreement with this hypothesis.

Our results show that the vascular perfusion density in the macular (SCP and DCP) and optic disc area was significantly decreased in RP patients while the FAZ area (SCP and DCP) was not significantly different from controls

Changes in the vasculature and haemodynamics of the retina have long been associated with RP. Ophthalmoscopically, attenuation of the retinal vessels is seen long before bone spicule pigment formation. In the pathology of eyes with RP, a reduction of the oxygen consumption due to photoreceptor cell loss has been suggested to cause oxygen diffusion from the choroidal vessels into the inner retina, which decreased the need for oxygen delivery 

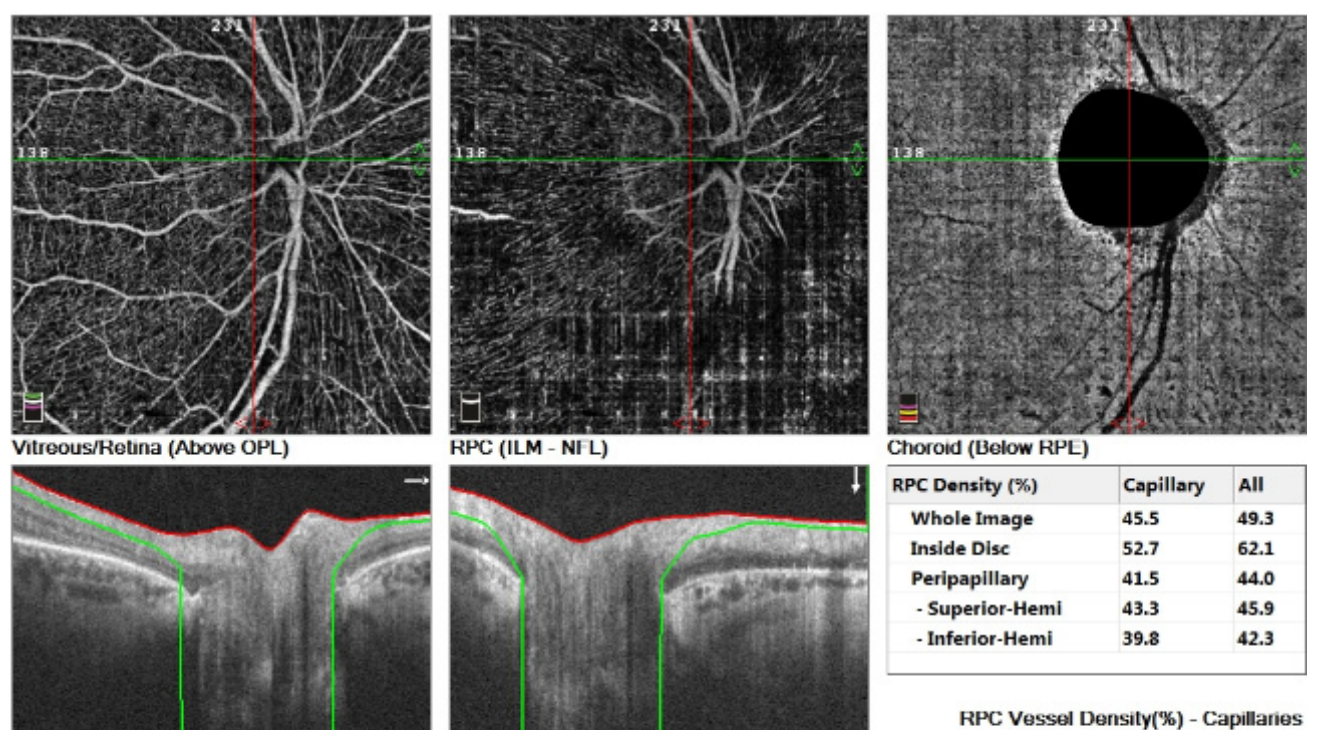

RPC (ILM - NFL)

Choroid (Below RPE)

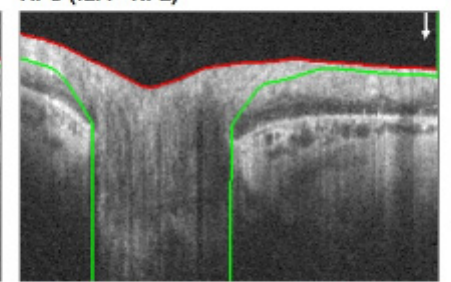

\begin{tabular}{|l|l|l|}
\hline RPC Density (\%) & Capillary & All \\
\hline Whole Image & 45.5 & 49.3 \\
Inside Disc & 52.7 & 62.1 \\
Peripapillary & 41.5 & 44.0 \\
- Superior-Hemi & 43.3 & 45.9 \\
- Inferior-Hemi & 39.8 & 42.3 \\
\hline
\end{tabular}
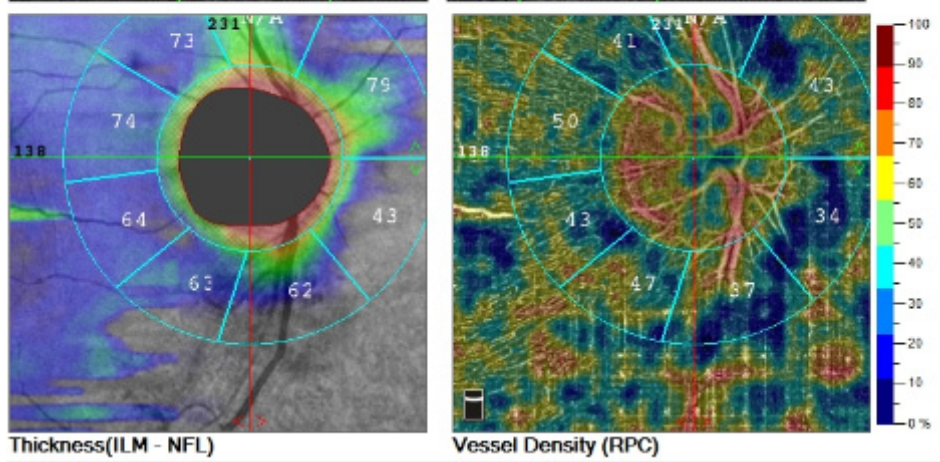

RPC Vessel Density(\%) - Capillanies

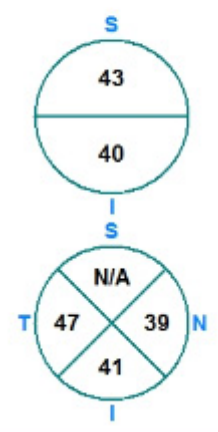

FIGURE 2. One of the patient's radial peripapillary capillary (RPC) vascular density map

from the retinal circulation; hence, oxygen diffusion was assumed to cause an attenuation of the vessels $[5,6]$. Other histopathologic studies, however, have revealed that the migration of retinal pigment epithelium (RPE) cells around inner retinal blood vessels stimulates deposition of extracellular matrix that resembles ectopic Bruch's membrane, and this perivascular matrix progressively thickens and occludes the lumen of the vessels, leading to compromised blood flow [7, 8].

Previous cross-sectional studies using spectral-domain OCTA with a $3 \times 3 \mathrm{~mm}$ image area showed that the choriocapillaris blood flow in patients with RP was not different from those of controls $[4,9]$. On the other hand, Miyata et al. demonstrated concentric and vermicular choriocapillaris flow deficits in patients with RP using wide-angle swept-source OCTA [10], which is a finding, according to them, that could not be detected by conventional angle OCTA with an image area of
$3 \times 3 \mathrm{~mm}$ because the maximum circle area detectable is smaller than the required area to detect these findings [10]; our study result is in general consensus with this study and does not show a significant difference between RP patients and controls.

Previous studies have also demonstrated reduced retinal blood flow in patients with RP using several techniques, including high-resolution magnetic resonance imaging (MRI), laser speckle flowgraphy and laser Doppler flowmetry [11]. These results correspond with the findings of the present study. Nevertheless, Toto et al. reported that choriocapillaris capillary plexus densities in patients with middle- and late-stage RP were significantly lower compared to those in control subjects. This reduction in choriocapillaris capillary plexus density is probably secondary to the stage of RP [12].

We found that the FAZ area was non-significantly larger in patients with RP than in controls ( $\mathrm{p}>0.05)$. Recent studies are not conclusive regarding the ex- 
pansion of the FAZ area in patients with RP. Some authors reported that the FAZ at the deep capillary plexus level was larger in patients with RP than in controls [4]. Other authors found no significant difference in the FAZ in both superficial and deep layers between patients with RP and controls [13].

We are aware of the limitations to this study, which include the small sample size. The main reason behind this is that OCTA is a relatively new device; hence, it is challenging to collect patients with longer follow-up who qualify for the study. Moreover, in order to produce an accurate analysis, only patients with high-quality OCTA scans were analysed. Due to the fact that the acquisition of high-quality OCTA images is heavily dependent upon the patient's ability to fixate, most patients with advanced RP were excluded from the study because they lacked fixation due to poor vision. This dramatically decreased the sample size (eight out of 80 patients) and limited the possibility of studying changes in the retinal and choroidal vasculature in patients with advanced RP, a stage at which one might observe significant changes.

\section{CONCLUSION}

Given the abovementioned results, we recommend using OCTA as an auxiliary test when approaching patients with RP

\section{REFERENCES}

1. Hartong DT, Berson EL, Dryja TP. Retinitis pigmentosa. Lancet. 2006; 368(9549): 1795-1809, doi: 10.1016/S0140-6736(06)69740-7, indexed in Pubmed: 17113430.

2. Grunwald JE, Maguire AM, Dupont J. Retinal hemodynamics in retinitis pigmentosa. Am J Ophthalmol. 1996; 122(4): 502-508, doi: 10.1016/ s0002-9394(14)72109-9, indexed in Pubmed: 8862046.
3. Coscas GJ, Lupidi M, Coscas F, et al. Optical coherence tomography angiography versus traditional multi- modal imaging in assessing the activity of exudative age- related macular degeneration: a new diagnostic challenge. Retina. 2015; 35(11): 2219-2228, doi: 10.1097/ IAE.0000000000000766, indexed in Pubmed: 26398697.

4. Battaglia Parodi M, Cicinelli MV, Rabiolo A, et al. Vessel density analysis in patients with retinitis pigmentosa by means of optical coherence tomography angiography. Br J Ophthalmol. 2017; 101(4): 428-432, doi: 10.1136/bjophthalmol-2016-308925, indexed in Pubmed: 27343210.

5. Eysteinsson T, Hardarson SH, Bragason D, et al. Retinal vessel oxygen saturation and vessel diameter in retinitis pigmentosa. Acta Ophthalmol. 2014; 92(5): 449-453, doi: 10.1111/aos.12359, indexed in Pubmed: 24767302.

6. Türksever C, Valmaggia C, Orgül $S$, et al. Retinal vessel oxygen saturation and its correlation with structural changes in retinitis pigmentosa. Acta Ophthalmol. 2014; 92(5): 454-460, doi: 10.1111/aos.12379, indexed in Pubmed: 24767408.

7. Li ZY, Possin DE, Milam AH. Histopathology of bone spicule pigmentation in retinitis pigmentosa. Ophthalmology. 1995; 102(5): 805-816, doi: 10.1016/s0161-6420(95)30953-0, indexed in Pubmed: 7777280.

8. Milam AH, Li ZY, Fariss RN. Histopathology of the human retina in retinitis pigmentosa. Prog Retin Eye Res. 1998; 17(2): 175-205, doi: 10.1016/s1350-9462(97)00012-8, indexed in Pubmed: 9695792.

9. Sugahara M, Miyata M, Ishihara K, et al. Optical Coherence Tomography Angiography to Estimate Retinal Blood Flow in Eyes with Retinitis Pigmentosa. Sci Rep. 2017; 7: 46396, doi: 10.1038/srep46396, indexed in Pubmed: 28406171.

10. Miyata M, Oishi A, Hasegawa $T$, et al. Concentric Choriocapillaris Flow Deficits in Retinitis Pigmentosa Detected Using Wide-Angle Swept-Source Optical Coherence Tomography Angiography. Invest Ophthalmol Vis Sci. 2019; 60(4): 1044-1049, doi: 10.1167/iovs.1826176, indexed in Pubmed: 30897619.

11. Takagi S, Hirami Y, Takahashi M, et al. Optical coherence tomography angiography in patients with retinitis pigmentosa who have normal visual acuity. Acta Ophthalmol. 2018; 96(5): e636-e642, doi: 10.1111/ aos. 13680, indexed in Pubmed: 29498230.

12. Toto L, Borrelli E, Mastropasqua R, et al. Macular Features in Retinitis Pigmentosa: Correlations Among Ganglion Cell Complex Thickness, Capillary Density, and Macular Function. Invest Ophthalmol Vis Sci. 2016; 57(14): 6360-6366, doi: 10.1167/iovs.16-20544, indexed in Pubmed: 27898981.

13. Koyanagi Y, Murakami Y, Funatsu J, et al. Optical coherence tomography angiography of the macular microvasculature changes in retinitis pigmentosa. Acta Ophthalmol. 2018; 96(1): e59-e67, doi: 10.1111/ aos. 13475, indexed in Pubmed: 28561452. 